$\begin{array}{ll}\text { le portiQue } & \text { Le Portique } \\ \text { Revue de philosophie et de sciences humaines }\end{array}$

$20 \mid 2007$

Gilles Deleuze et Félix Guattari : Territoires et devenirs

\title{
Ce que fait Deleuze à la danse
}

\section{Roland Huesca}

\section{OpenEdition}

\section{Journals}

\section{Édition électronique}

URL : http://journals.openedition.org/leportique/1368

DOI : $10.4000 /$ leportique.1368

ISSN : $1777-5280$

\section{Éditeur}

Association "Les Amis du Portique"

Édition imprimée

Date de publication : 15 décembre 2007

ISSN : 1283-8594

\section{Référence électronique}

Roland Huesca, «Ce que fait Deleuze à la danse », Le Portique [En ligne], 20 | 2007, mis en ligne le 06 novembre 2009, consulté le 26 mars 2021. URL : http://journals.openedition.org/leportique/1368 : DOI : https://doi.org/10.4000/leportique.1368

Ce document a été généré automatiquement le 26 mars 2021

Tous droits réservés 


\title{
Ce que fait Deleuze à la danse
}

\author{
Roland Huesca
}

1 Aujourd'hui, «l'air du temps » aime s'inspirer de Gilles Deleuze, de ses écrits, de sa pensée, de ses concepts. Dans le domaine des arts, les idées du maître prennent chair pour devenir couleur, odeur, mouvements. Pour autant, comme le rappelle Anne Cauquelin, il ne s'agit pas pour ces artistes de faire de ces concepts les dérivés de sensations, ni, à l'inverse, de traduire les œuvres en terme de raison. Mais de s'inspirer de cette philosophie de l'art et de la vie pour appréhender le monde de la création. De l'accompagner pour parfois l'accommoder ou s'en détourner : «Ainsi, le concept quitte son ancrage, emprunte l'allure de la couleur et du son, se met en mouvement et produit du mouvement ${ }^{2} »$.

2 Dans cet univers, depuis les années quatre-vingt-dix, certains textes de Gilles Deleuze ont animé, et légitimé, les œuvres d'une poignée de chorégraphes situés à la marge de leur art. Véritables ressources cognitives disponibles et utilisables à loisir, des bribes de sa pensée ont offert à certaines créations et réceptions artistiques un ancrage culturel socialement et solidement construit. Sans induire pour autant un discours de vérité, ces emprunts ont cristallisé une éthique ${ }^{3}$ minimisant le plus souvent la prégnance des universaux et des idéaux édictés par les récits modernistes. Dès lors, au creux des corps, déconstruisant les croyances les plus communes, certains savoirs ont engendré des savoir-faire artistiques.

3 À partir d'une analytique de la création, nous étudierons la morphologie et les enjeux de ces scènes esthétiques et discursives pour montrer comment et pourquoi, dans le milieu chorégraphique français, certaines références deleuzienne, qu'elles soient implicites ou explicites, sont devenues à la fois source d'inspiration et espace de commentaires. Cette analyse permettra alors de saisir comment et pourquoi ces diverses mises en œuvres ont déjoué les représentations du corps les plus communément admises. 


\section{Flux. 1 ère période}

4 1983. Au Théâtre de la Ville, une œuvre enthousiasme le public comme la critique. Hervé Diasnas propose son solo, Naï ou cristal qui songe ${ }^{4}$. Déjà, le titre promet pureté et poésie. Très vite la nouvelle circule dans les studios de danse parisiens. Il faut aller voir cet artiste d'exception. Son approche semble neuve. La scène s'allume. Apparaît un homme immobile. Sur son torse nu, un bâton. L'éclairage savamment étudié met en relief la beauté de cette nudité apollinienne. Délicatement le thorax se lève, puis s'affaisse. Lente et profonde, cette respiration dévoile la vie et instaure un lien entre le danseur, l'espace et le public. Dans ce va-et-vient primordial, l'inactivité supposée devient condensation de mouvement. Lentement, l'homme et son «bois » développent l'effet. La main se rapproche, glisse doucement le long de l'objet. Connivence. Loin de toute fixité apparente, cet effleurement impose un style. À terre, le travail prolonge l'impression. L'interprète évolue, sans bruit. Lorsque le corps allongé roule sur le côté, la genèse du geste imprime déjà sa marque au futur trajet. Le bassin lance son entame, les extrémités du corps suivent, comme à la traîne. L'artiste dessine de grands cercles dont il n'est que partie. Si le mouvement change de sens, l'elliptique s'inverse, mais toujours l'onde serpente. L'œil du public ne perçoit jamais la présence de quelque chose de fixe. Tout se passe dans la mouvance, l'instabilité perpétuelle. Inscrit dans un flux, l'interprète déploie sa danse. Sans abandonner pour autant, la dynamique du geste supplante la forme. Au quotidien, la pratique indique le chemin. Au sol, couché sur le dos, le danseur cherche à diffuser les battements de son cœur, le cycle de sa respiration. Peu à peu, il sentait le flux s'écouler dans les veines, parcourir sa chair ; peu à peu, la qualité de la propagation s'affinait. Sollicitant l'imaginaire, l'ouverture du corps était déjà un appel à la mouvance.

5 À ce moment, le mouvement ne se définit plus en fonction d'un point d'origine, d'une zone de fixité, mais se fond dans le flux qui le traverse ${ }^{5}$. Dans cette synergie d'appuis mouvants, la trajectoire prime le point, et la durée l'instant. Dans les années quatrevingt, ce souffle inédit s'inspire de l'air du temps. Selon Gilles Deleuze ${ }^{6}$ à partir des années quatre-vingt, les pratiques d'exercice corporel, à l'image du surf, délaissent les actions requérant des appuis et des bras de levier pour s'insérer dans le trajet d'une onde préexistante. Se mettre en orbite, se faire accepter dans le mouvement d'une grande vague exprime une nouvelle modalité d'être. Désormais, l'homme aime onduler sur des faisceaux continus, qu'ils soient imaginaires ou réels. Adepte du Tai-chi, Hervé Diasnas incorpore cet imaginaire. À sa manière, il rencontre les problématiques anciennes pour qui dans « l'air infini..., s'effacent les dimensions ${ }^{7}$ » du corps. Mais à la différence du projet bachelardien, au final, ici, pas de mise en retrait de la corporéité au profit d'une spiritualité contemplative. Au contraire, dans la lignée de Gilles Deleuze, avec lui la chair se place au centre de l'expérience et, en elle, cherche à se magnifier.

\section{Flux. $2^{\mathrm{e}}$ période}

6 1996, Boris Charmatz crée Aatt Enen Tionon au théâtre de la Halle aux Grains à Blois ${ }^{8}$ Une structure verticale à trois niveaux accueille les danseurs : en bas, le chorégraphe, tout en haut, Julia Cima, au milieu Vincent Druguet. Ouvert aux regards, ce «bloc chorégraphique ", haut de cinq mètres environ, reste exigu : deux mètres sur deux. Dans cet espace érigé, le trio prend place et s'échauffe; alentour, le public déambule, 
observe et s'interroge sur ce dispositif peu banal. Ne gardant en tout et pour tout qu'un seul tee-shirt, les artistes se dévêtent. Le spectacle commence. Verges, vulve, fesses, immanquablement, en un puissant corps à corps, ces nudités exposées parlent crûment à la nôtre.

7 Se démarquant du «bien fait et de l'agréable " ${ }^{9}$, les danseurs explorent des gestes spasmodiques, agités et sans cesse brisés. Loin d'une esthétique de la grâce, vantant la gestuelle fluide et arrondie chère au monde chorégraphique, loin aussi des verticalités mise en œuvre dans le ballet, leurs danses faites de heurts, de soubresauts, de chutes, d'immobilités contenues et de tensions les arrachent de représentations idéelles et utopiques des corps mise en scène par la modernité de la Belle époque. Dans cette structure de métal, ces esseulements relatifs déjouent les conventions. Pas de mouvement d'ensemble, pas de gestes codifiés permettant au spectateur d'anticiper le perçu ou de faire disparaître l'œuvre derrière une image idéale. Seulement des corps en action ramenant toujours le vu au visible, et un dispositif obligeant le public à choisir son voir, à lier la danse à l'actualité de visions résolument singulières.

Devant Aatt Enen Tionon, le dramaturge Joris Lacoste lance sa mise en garde : "Car les orifices du corps ainsi mis à nu, il est facile de pressentir que tous les flux bouche-anussexe, littéralement et dans tous les sens, sont à l'œuvre. Que c'est par là que passe la danse. Qu'il y a un affût des corps entre eux et que c'est cette tension même qui fait advenir quelque chose ${ }^{10}$. Au fil des mots, nous voici en compagnie de Gilles Deleuze et de son ami Félix Guattari ${ }^{11}$. De leurs écrits, ces auteurs avaient ouvert un chemin : « Un organe peut être associé à plusieurs flux d'après des connexions différentes; il peut hésiter entre plusieurs régimes, et même prendre su soir le régime d'un autre organe [...] ${ }^{12}$ ». Dans le monde de l'art, souvent, la puissance métaphorique de cette rhétorique savante organise ou conforte, çà et là, les dires et les faire de ces artistes de la marge. Touchant justes, les mots font sens car ils trouvent un écho dans l'imaginaire des artistes. Champs de forces, flux, dans les livres ou sur les scènes, ces images dynamisent et enrichissent les représentations de la corporéité. Au creux des chairs, les "états intensifs » des corps dansants détruisent l'unité fictive d'un moi idéal ${ }^{13}$. Mettant en œuvres ses visions du monde, le danseur ne cherche pas «le » corps dans le corps. Il ne veut pas rendre claires ses significations obscures, mais désire simplement explorer le dépouillé de la chair sur lequel s'incarnent des corporéités historiquement déterminées, et, de fait, relatives et contingentes. Car si ces peaux exhibées portent en elles une vérité du corps, elles dénient à chacune d'elles la capacité d'incarner le corps en sa vérité. À l'encontre des absolus idéalisant l'individu en une totalité, elles le dévoilent comme conscience d'être soi, individué et unique.

Ouvrir des brèches dans l'ordre des certitudes, penser autrement le visible, montrer le caractère non maîtrisable des choses, témoigner de leur infinitude, présenter l'animalité d'êtres littéralement "à poil », provoquer l'intime et les sens: dans la gestion des flux du corps, le projet artistique prend vie dans l'intensité du geste.

D'Hervé Diasnas à Boris Charmatz, du torse au cul, du souffle à l'intensité, tout un ensemble de représentations liées à la corporéité bascule dans cette danse des flux.

\section{Territoires}

11 Ce soir on donne Jérôme Bel, œuvre éponyme de l'artiste. [...] Placée à l'avant-scène, la danseuse s'empoigne sous la poitrine, tire sa peau et la relève sur ses seins. Le danseur 
la rejoint, attrape l'épiderme de ses testicules, le remonte et masque sa verge... Dans le fragment de cette signification commune où s'effacent les marques de la sexualité, les deux corps entrent en relation... La femme retourne en fond de scène. Lâchant sa prise, l'homme porte sa main à la bouche, la mouille et dirige sa paume vers la cuisse. D'un geste circulaire et rapide, il se frotte les poils, puis retire sa main... Et voilà la zone pileuse transformée en un amas disparate de petites pelotes velues... Juste au-dessus de cette surface, l'artiste pointe un grain de beauté, puis un autre... Obéissant au doigt et à l'œil, la vision du public peine à distinguer le vu du visible. Bien vite, à l'image des pigments mis à l'index, les boules de poils se font nævi... La femme revient, sort de sa bouche un bâton de rouge à lèvres [...] dessine un cercle autour de sa taille, puis un autre autour de sa poitrine, se tourne et amorce, sur le milieu du dos, une verticale partant du trait inférieur. L'homme la retrouve, prend le bâton, et délicatement termine le segment jusqu'à l'autre trait... En un éclair, ce geste transforme les lignes en bustier dont on relève la fermeture [...].

Voici comment le chorégraphe Alain Buffard commente Jérôme Bel dans la revue Mouvement: "...chaque inscription, chaque marquage, chaque surlignage des corps, dans Jérôme Bel, traduit une valeur propre, autonome, étoilée par des réseaux d'interprétations connexes ${ }^{14}$. Comme son pair, Alain Buffard aime articuler sa pensée en référence à Gilles Deleuze. Sans prétendre à une quelconque exégèse des textes du philosophe, ces deux créateurs s'enrichissent de cette pensée complexe, spatialisée au gré des métaphores et faisant la part belle à une esthétique de la sensation. En percevant des "réseaux d'interprétations connexes ", le commentaire d'Alain Buffard porte l'empreinte de cette affinité élective. À l'image de l'univers rhizomatique développé par Gilles Deleuze et son ami Félix Guattari, Jérôme Bel met en évidence une coexistence multiple de territoires distincts présentés puis dérobés. Sans cesse, l'artiste se défait des intentions premières, sans cesse, il habille la nudité d'un autre effet. En d'habiles concordances, le corps devient un lieu de passages et de métamorphoses, un carrefour de significations singulières et contiguës, construisant et déconstruisant sans relâche les signifiants proposés.

13 En prenant pour fil conducteur la trame deleuzienne, un observateur averti peut, à chaque instant, saisir comment le danseur fait et défait un espace de corps. Devenue lieu commun, la peau relevée sur les marques sexuelles se mue en surface de passagepour faire le lien entre deux instants chorégraphiques, là, la boule de poils devient grain de beauté, plus loin, le trait se transforme en corset. Véritables charniers de signes, chaque surface corporelle s'orne sans cesse des traces d'un référent culturel, puis l'annihile par la mise en présence d'un autre contexte. Inéluctablement, le geste, le mot ou le tracé farde le tégument, le déguise à chaque fois d'une vérité différente. Car ici, la nudité ne saurait être vierge de tout artifice. Quittant un territoire pour un autre, et multipliés à l'envi, des territoires corporels apparaissent puis se dérobent. L'équation signe-nudité devient un simple effet de langage, un précipité toujours réinventé de l'ordre sémiotique. À même la peau, ces diverses déconstructions injectent leur relativisme épistémologique et axiologique. Admettant pour trame la conjonction « et... et... et... ${ }^{15}$, chaque espèce d'espace déploie ses ramifications; "la multiplicité se métamorphose en changeant de nature ${ }^{16}$. À l'image de l'énoncé deleuzien, l'œuvre élabore une dialectique de singularités multiples. Loin des conceptions univoques du monde, elle accepte la coexistence d'univers singuliers au sein d'un même plan de 
consistance. Du même geste, le pluriel se substitue au singulier. Évanoui, l'idéal du corps ne fait plus figure d'autorité.

Au cœur de ces soirées à fleur de peau, l'intelligible excède le sensible pour mettre en rapport des faits et gestes isolés. Sur scène, le corps à corps danseurs-public propose un ailleurs, plus notionnel, incitant à un acte d'intellection complexe très éloigné des seules expériences perceptives élémentaires. Cependant, Arthur Danto avait lancé sa mise en garde. Pour s'exercer, la fonction rhétorique des œuvres conceptuelles doit offrir aux spectateurs les clés de la compréhension. Dans le cas contraire, " on ne saurait en ressentir la puissance $"{ }^{17}$. Conscients du fait, les danseurs inventent parfois des modes de sociabilités originales. Après certaines de leurs pièces, Alain Buffard ou Xavier Le Roy organisent un débat avec la salle. Le temps est à l'échange. Ailleurs, ces mêmes artistes noircirent les colonnes de la revue Mouvement sur et pour Jérôme Bel. Plus tard, Art Press sert de tribune à Jérôme Bel pour évoquer le travail de Xavier le Roy, ${ }^{18}$ dont l'œuvre Gizselle repose sur un principe identique à celui animant Jérôme Bel. Amis, spectateurs et membres d'un même clan, ces chorégraphes, et c'est nouveau, opèrent leurs critiques non pas dans des revues spécialisées sur la danse, mais dans des feuilles vouées à l'art contemporain. À la manière "d'une société savante», ils exposent, commentent et expliquent quelques-unes de leurs propositions artistiques. Ici, ce danser nu ne cherche pas « Le » corps dans le corps. Il ne veut pas rendre claires ses significations obscures, mais explore simplement le dépouillé sur lequel s'incarnent des corporéités historiquement déterminées, et, de fait, relatives et contingentes. Et si ces peaux exhibées portent en elles une vérité du corps, elles dénient à chacune d'elles la capacité d'incarner le corps en sa vérité.

15 Intuitive, liée au corps de façon immanente, la danse restait une pratique de la marge. Longtemps dominée par les autres arts, elle trouve, dans le jeu puissant des mots, l'occasion de convoiter des espaces identiques, de hanter des lieux de discours analogues. En se liant à une rhétorique postmoderne, elle gagne une part de légitimité. Jusqu'ici, peinture et musique en tête, la regardaient de haut. De la profusion à la rareté, un rapide coup d'œil sur les étales des librairies confirme la tendance. Prenant le corps pour objet d'étude, ces conceptuels travaillent la distinction.

\section{Figures}

16 À partir des années 1990, comme Xavier Le Roy, quelques danseurs exposent sur scène leur étrange nudité. Virtuoses de la lenteur et de la contorsion, ils présentent et renouvellent sans cesse, une multitude de formes nouvelles et inédites. De pose en pose, ils défigurent le corps pour mieux le ré-envisager. Voici comment, dans les colonnes de Art Press, le danseur chorégraphe Jérôme Bel commente Self-Unfinished, l'œuvre de Xavier Le Roy : «Ainsi, en se mettant littéralement "cul par-dessus tête", le plus trivialement du monde, qu'il crée des formes tout à fait étonnantes avec pour seul outil, j'insiste, son corps. Ces formes sont distillées avec une lenteur calculée, sans changement apparent parfois. Ces formes me sont apparues, et là je dois parler de mon expérience personnelle de spectateur, monstrueuses. J'y ai vu un homme sans tête, une tête d'éléphant, un poulet prêt à rôtir, un batracien, un phallus, plusieurs sortes d'extra-terrestres, un escargot, une danseuse qui fait le grand écart...Tout cela n'a rien de monstrueux en tant que tel, cependant, ces images apparaissent alors que le corps de Xavier Le Roy, ne bouge pas ou se déplace de manière tout à fait limitée sur la scène. 
Aucune lumière particulière ni aucun autre illusionnisme n'opèrent ici. Il est seul au milieu de la scène, nu sous des néons blafards ${ }^{19}$ ». Sur le plateau, à même la peau, de multiples représentations du corps se succèdent. À la lueur d'un seul effet de lumière des néons blancs donnant au cadre de scène des allures de "white cube " - l'artiste déploie ses contorsions étranges et disparates. Faisant la part belle à la forme et à ses singularités multiples, ce lieu monochrome suspend les imaginaires les plus communs ; sa neutralité déjoue les inférences et les effets de contexte pouvant renforcer la part humaine lovée en chacun. L'heure est au bizarre, à une esthétique de la dissemblance et de l'écart entre les représentations normatives du corps humain et l'objet/corps présenté. Dans la mise en jeu lente de ses images, l'artiste s'émancipe des représentations les plus conformes. Les incessantes métamorphoses de ces figures plurielles valorisent chacun des lieux où elles se stabilisent.

Pour rendre compte de son œuvre, Xavier le Roy, comme ses pairs chorégraphes, Maria Donato d'Urso, Benoît Lachambre, etc., ne cachent pas leurs emprunts aux savoirs et savoir-faire des arts visuels. Notamment aux techniques employées par Laurent Goldring, créateur avec lequel ils ont collaboré en posant nus devant sa caméra. Façonnant des formes corporelles improbables sur les conseils et les recommandations du plasticien, ils conçoivent leurs corps comme une masse plastique à construire et à déconstruire. Dans le labeur du studio, le processus créateur convoque à la fois la proprioception du danseur, ses sensations, ses états intérieurs, mais aussi un regard extérieur matérialisé par l'image vidéo et garant de la genèse de l'œuvre. Multipliant les "figures", les membres et le tronc, s'unissent, se dissocient, s'assemblent autrement pour composer d'étranges scènes abstraites et mouvantes ${ }^{20}$. Sans visage et sans sexe, donc sans identité préétablie, ces formes n'aspirent à une quelconque identification. Il n'est pas question de reconnaître, un bras un pied, une fesse, un homme ou une femme: «Le corps représenté doit en passer par des intensités d'existences qui sont en même temps des déformations, des protomouvements qui les portent ${ }^{21}$ ». Au bout du regard et sur l'écran, tout n'est plus que fragments, masses et lignes, et mise en circulation de variations de tensions et de forces.

Mais pourquoi, à ce moment, ces références et ce type de travail? Passionné de philosophie et d'histoire de l'art, Laurent Goldring déploie son œuvre en dialoguant avec les auteurs qui animent sa pensée. Parmi eux, Gilles Deleuze et ses écrits sur le cinéma et sur Francis Bacon notamment. Ce qu'il en retient? L'idée de se défaire des représentations normatives de la corporéité, de leurs usages et de leurs significations les plus évidentes. Naît alors, le désir de créer des matières a-signifiantes et asyntaxiques, mais esthétiquement et pragmatiquement formées. Au chevet de ces corps/matière, l'artiste met en œuvre des formes nouvelles et inédites en exposant des forces, des attentes et des poussées du corps. Bref, il met en scène des « Figures » dans le sens deleuzien du terme. Cette approche de l'art privilégie le les affects réels ${ }^{22}$. Factuelle et réaliste, elle répond à une logique de la sensation perçue dans l'immanence et non à un ensemble de représentations préétablies. Sans se soucier d'une quelconque figuration, l'artiste cherche à capter le moment ou quelque chose passe, ou quelque chose a lieu, dans le surgissement sensible de la Figure ${ }^{23}$.

19 Pour ces artistes, chorégraphes et plasticiens, les références deleuziennes participent d'un moment mettant en avant la déconstruction du statut ontologique de l'image. Au cœur de la dissemblance, elle cherche à montrer combien l'identité n'est pas un en-soi, mais doit se percevoir comme une multiplicité quasi infinie de formes où les modalités 
du paraitre s'organisent à partir d'une matrice produisant des variations de figures. Bigarrée, l'identité se met alors en scène comme le mouvement des identités multiples. Dès lors, le corps devient le lieu d'une pensée agile défiant les "prévisions " d'une raison aimant construire les effets de sens à partir d'a priori culturellement définis et révélant, trop souvent, l'incapacité de l'Homme occidental à accepter l'évidence de niveaux d'existence différents au sein d'un même être.

\section{Conclusion} rhétorique deleuzienne, artistes et commentateurs ne se sont pas bornés à appliquer une vue de l'esprit; dans leurs œuvres ils ont sélectionné, intériorisé, modifié et bien sûr incorporé ces énoncés, parfois même inconsciemment. Il faut dire que, dans les dédales du vingtième siècle, les multiples "déconstructions " ont mis en crise les usages. Du même geste, ce mouvement de pensée a transformé le corps en une véritable réserve de sens donnant un souffle nouveau à l'art et à la pensée.

\section{NOTES}

1. . Une conception du monde au sens de Max WEBER, L'Éthique protestante et l'Esprit du capitalisme, 1904-1905, Paris, Plon, 1964.

2. . Anne CAUQUELIN, «Présentation », Revue d'esthétique n 45, décembre 2004, p. 5-7.

3. . Une conception du monde au sens de Max WEBER, L'Éthique protestante et l'Esprit du capitalisme, 1904-1905, Paris, Plon, 1964.

4. . 1983.

5. . La danse moderne aux États-unis et en Allemagne, puis dès les années cinquante, la danse contemporaine avec Merce Cunningham notamment, avaient jeté les bases d'un renouveau. Cependant, dans les techniques de corps les vigilances centrées sur la fixité de l'appui guidaient le faire.

6. . Gilles DELEUZE, Pourparlers, Paris, Minuit, 1990, p. 165.

7. . Gaston BACHELARD, L'Air et les Songes, Paris, Corti, 1943, p. 17.

8. . La pièce sera reprise au festival d'automne à Beaubourg la même année.

9. . Boris ChARMATZ, « Le choix du Pire », Mouvement, octobre 1996.

10. . Joris LACOSTE, «La nudité froide de Boris Charmatz », Le Journal de l'ADC, Genève, $\mathrm{n}^{\circ}$ 14, avril 1997, p. 8.

11. . Mais aussi de Jean-François Lyotard.

12. . Gilles Deleuze et Félix GUATTARI, L'Anti-@Edipe. Capitalisme et schizophrénie, Paris, Minuit, 1972/1973, p. 46. 
13. Ibid., p. 102.

14. . Xavier LE ROY et Alain BUfFARD, «Dialogue sur et pour Jérôme Bel », Mouvement $\mathrm{n}^{\circ}$ 5, juin/ septembre 1999, p. 29-31.

15. . Gilles DeleuZe et Félix GUATTARI, Mille Plateaux, Paris, Minuit, 1980, p. 36.

16. Ibid., p. 32 .

17. . Arthur DANTO, La Transfiguration du banal une philosophie de l'art, 1981, Paris, Seuil, 1989, p. 275.

18. . Jérôme BEL, « Qu'ils crèvent les artistes ! ", Art Press n 23 spécial, 2002 ; p. 92-96.

19. . Ibid., p. 92-96.

20. . Programme théâtre de Cornouaille, Scène nationale de Quimper, saison 2004-2005.

21. . Laurent GOLDRING, «Entretien avec Laurent Goldring : propos recueillis par Cyril Béghin et Stéphane Delorme », Balthazar n 5, mars 2002.

22. . Anne sauvagnargues, « De la capture de forces à l'image ", Revue d'esthétique $\mathrm{n}^{\circ} 45$, décembre 2004, p. 51-66.

23. . Ibid.

\section{RÉSUMÉS}

Flux, territoires, figures: à la fin du xxe siècle, les bribes d'une rhétorique deleuzienne envahissent la sphère de certains chorégraphes situés à la marge de leur art. Sans induire pour autant un discours de vérité, ces emprunts ont cristallisé une éthique ${ }^{1}$ minimisant le plus souvent la prégnance des universaux et des idéaux édictés par les récits modernistes.

What Deleuze does at danse Flux, territories, figures - at the end of the 20th century, some choreographers, who don't belong to the main tide, have used excerpts from Deleuze's rhetoric in their work. These borrowings don't infer any attempt at a truthful discourse, yet an ethic has stemmed from them. Most of the time, it belittles the modernistic ideals.

Was Deleuze dem Tanz antut Fluxus, Territorien, Figuren: am Ende des 20. Jahrhunderts überfluten Bruchteile der deleuzischen Rhetorik die Welt einiger am Rande ihrer Kunst situierten Choregrafen.

\section{AUTEUR}

\section{ROLAND HUESCA}

Roland Huesca est Maître de conférences habilité à l'Université Paul Verlaine-Metz. Membre du laboratoire ERASE 2L2S, ses travaux actuels portent sur les rapports entre art et vieillesse et art et nudité. 